\title{
Schützenswerte seltene Baumarten im Randbereich des Botanischen Gartens Frankfurt
}

Hans GRASMÜCK

\begin{abstract}
Since 2012, the Botanic Garden belongs to the Palmengarten owned by the city of Frankfurt, whereas the neighbourhood area with former institutional buildings is possessed by the country of Hessia. The area hosts several old trees that should be protected if the area is used for other purposes in future.
\end{abstract}

\section{Zusammenfassung}

Seit 2012 gehört der Botanische Garten zum Palmengarten der Stadt Frankfurt, wohingegen das benachbarte Grundstück mit den ehemaligen Institutsgebäuden dem Land Hessen gehört. Auf diesem Grundstück stehen auch einige wertvolle Gehölze, die bei weiteren Planungen berücksichtigt und nach Möglichkeit erhalten werden sollten.

\section{Einführung}

Seit 1.1.2012 ist der Botanische Garten Frankfurt mit dem Palmengarten vereinigt. Daraus haben sich die Besitzverhältnisse der Grundstücke verändert, die auf dem Vertrag vom 3. September 2010 zwischen dem Land Hessen und der Stadt Frankfurt beruhen. In diesem Vertrag ist u.a. geregelt, dass die ehemaligen Institutsgebäude („Kramerbauten“) und enger anschließende Flächen beim Land Hessen verbleiben und nicht mehr unter die Zuständigkeit der
Universität Frankfurt fallen. Es wird sich vermutlich in der näheren Zukunft zeigen, wie diese Gebäude und Flächen genutzt werden.

In Abhängigkeit von der zukünftigen Nutzung der Fläche und den bestehenden Möglichkeiten bei der Realisierung sollten die wertvollen Bäume mitberücksichtigt und nach Möglichkeit geschützt werden. Dieser mehrere Jahrzehnte alte Baumbestand hat positive stadtklimatische Bedeutung, besonders auch im Kontext mit einem im benachbarten Grüne-

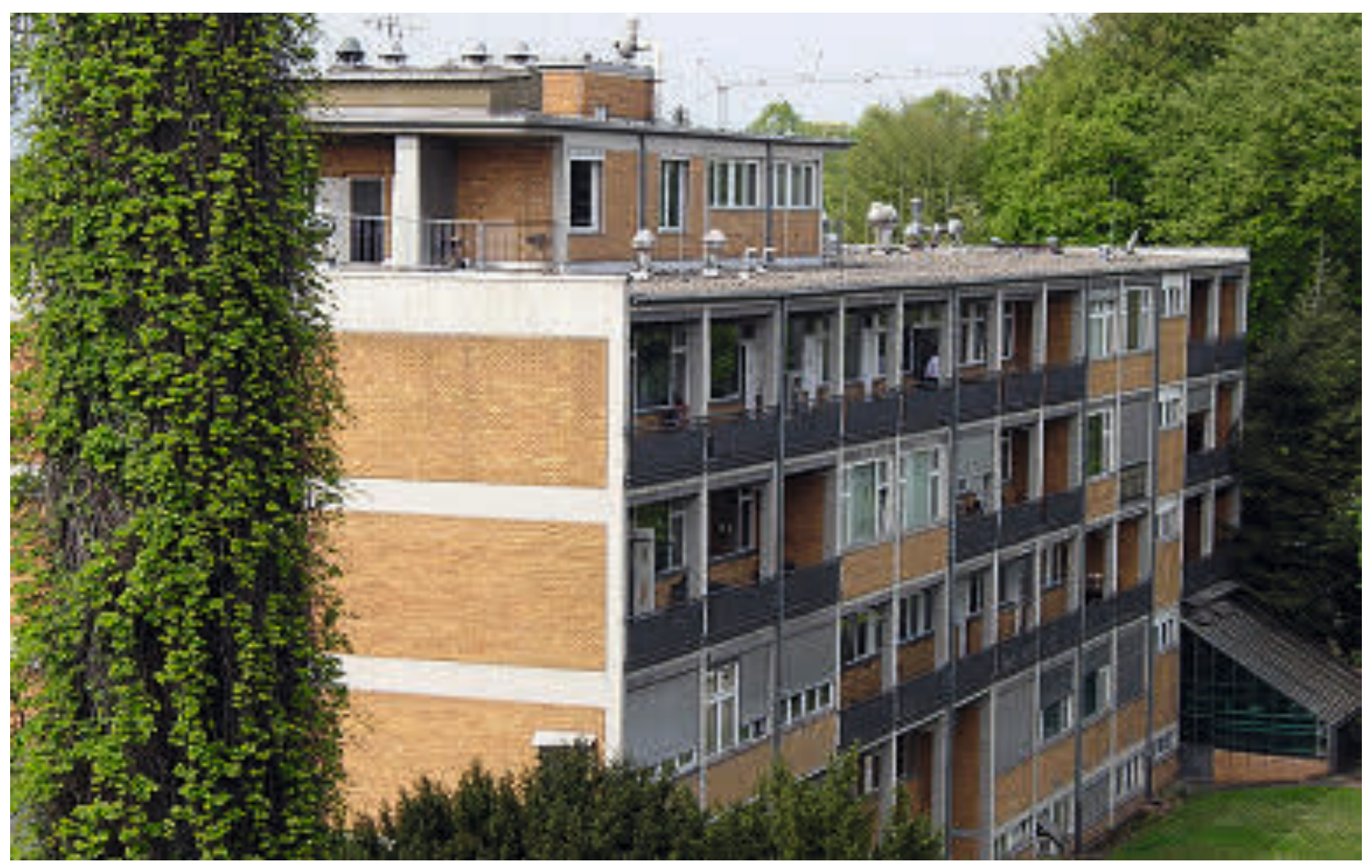


burgpark unmittelbar angrenzenden Gehölzbestand.

Die meisten Bäume im südöstlichen Randbereich des Botanischen Gartens wurden in den Jahren 1966 bis 1975 gepflanzt. Alle Bäume sind gesund und befinden sich in einem sehr guten Zustand. Mittlerweile sind einige von ihnen zu recht stattlichen Exemplaren herangewachsen, wobei zahlenmäßig die Nadelbäume überwiegen. Die schützenswerten Gehölze im Randbereich des Gartens sollen im Folgenden vorgestellt werden.

\section{Laubbäume}

Die Amerikanische Platane (Platanus occidentalis, Wuchshöhe: $17,5 \mathrm{~m}$ ) ist eine der größten Laubbaumarten des östlichen Nordamerikas und kann an günstigen Standorten 50 m Höhe erreichen bei einem Stammdurchmesser von ca. $4 \mathrm{~m}$. Am natürlichen Standort kommt sie in Auwäldern in Höhenlagen unter $1000 \mathrm{~m}$ vor. Die drei- bis fünflappigen weinlaubartigen Blätter werden $22 \mathrm{~cm}$ lang und $25 \mathrm{~cm}$ breit. Die Fruchtstände stehen einzeln, nur selten zu zweit an den Zweigen. Die rissig gefurchte Borke blättert in kleinen Schuppen ab. Die Amerikanische Platane wird in Mitteleuropa deutlich seltener kultiviert als die Morgenländische (Platanus orientalis) und die Ahornblättrige Platane ( $P . \mathrm{x}$ acerifolia $=P . \mathrm{x}$ hispanica $)$.

Die Mongolische Linde (Tilia mongolica, Wuchshöhe: $14,5 \mathrm{~m}$ ) ist eine kleine Baumart, die in nur wenigen Botanischen Gärten zu finden ist. Sie ist in der Mongolei und in Nordchina heimisch. Hier wird sie gut $10 \mathrm{~m}$ hoch. Ihre Zweige sind leicht überhängend und braunrot gefärbt. Ihre glänzenden Blätter, die sich im Herbst goldgelb verfärben, sind tief gelappt und am Rande gesägt. Im Austrieb sind sie rötlich und vergrünen später. Sie erinnern an ein Weinblatt.

Die Holländische Linde oder auch HybridLinde (Tilia $x$ vulgaris, Wuchshöhe: $20 \mathrm{~m}$ ) ist eine bis $40 \mathrm{~m}$ hoch werdende Hybride zwischen Sommer- und Winter-Linde. Sie ist raschwüch-

Abb. 1 (Seite 22): Blick über das alte Institutsgebäude. sig und wird häufig als Stadt- und Straßenbaum gepflanzt. Hier gehört sie zu den frühesten Anpflanzungen im Zuge der Institutsneubauten im Jahre 1956.

Die Hubei-Stinkesche (Tetradium daniellii $=$ Euodia hupehensis) steht in der Nähe der Grenze zum Grüneburgpark. Dieser Baum ist in den Bergwäldern Nordchinas und Koreas heimisch und wird dort $15 \mathrm{~m}$ hoch. Stinkeschen werden bei uns selten außerhalb von Botanischen Gärten gepflanzt. Wegen ihrer reichen Sommerblüte ist die Stinkesche eine wertvolle Bienenfutterpflanze. Die kleinen, weißen, duftenden Blüten sind zu mehreren in breit-pyramidalen Rispen zusammengefasst. Auch die dunkel gefärbten Früchte tragen zum dekorativen Wert dieses Gehölzes bei.

Der Amerikanische Amberbaum (Liquidambar styraciflua, Wuchshöhe: $11 \mathrm{~m}$ ) wächst vor dem ehemaligen Tierstallgebäude. Der Baum wird auch American Sweetgum genannt. In seiner Heimat, den Auwäldern Nordamerikas, erreicht er eine Wuchshöhe von $45 \mathrm{~m}$. In Mitteleuropa wird er selbst an günstigen Standorten aber meist nur bis $20 \mathrm{~m}$ hoch. Der attraktive Zierbaum hat tief gelappte, ahornähnliche, aber wechselständig angeordnete Blätter mit gelber, scharlachfarbener bis weinroter Herbstfärbung. Beim Zerreiben duften die Blätter aromatisch süßlich. Die gestielten, verholzten, kugeligen Kapselfruchtstände erinnern in ihrem Aussehen an kleine Seeigel und verbleiben oft den Winter über am Baum.

Von der Japanische Kornelkirsche (Cornus officinalis) gedeihen hier mehrere strauchförmig entwickelte Exemplare, die der bei uns heimischen Kornelkirsche (Cornus mas) sehr ähnlich sind. Von dieser unterscheidet sich $C$. officinalis durch den Blütezeitpunkt, kleinere Früchte, bestimmte Blattmerkmale, Herbstfärbung sowie Struktur der Borke. Heimisch ist Cornus officinalis in China und Korea, in Japan ist sie seit langem eingeschleppt.

\section{Nadelbäume}

Der Küstenmammutbaum (Sequoia sempervirens, Wuchshöhe: $28,50 \mathrm{~m}$ ) gehört zu den eindrucksvollsten und leistungsfähigsten Bäumen 


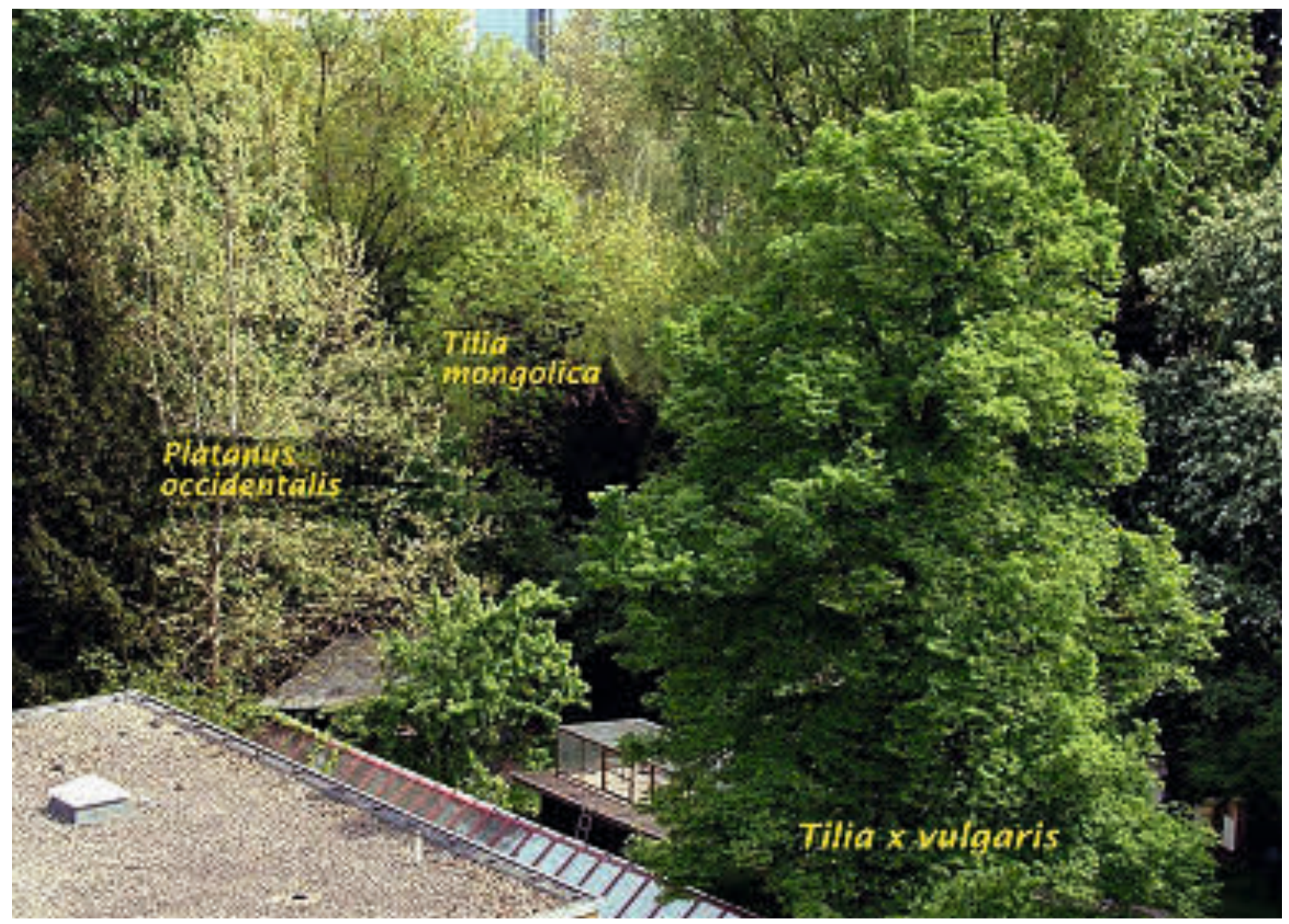

der Nordhalbkugel. Am Naturstandort, im Nebelgürtel der nordkalifornischen Pazifikküste, hat ein Exemplar 120 m Höhe, 4,50 m Stammdurchmesser und ein Alter von 2000 Jahren erreicht.

Auf dem Gelände stehen drei Exemplare der Nordmann-Tanne (Abies nordmanniana, geschätzte Wuchshöhen: 17, 18, und 21,5 m). Die Nordmann-Tanne ist im westlichen Kaukasus und der NW-Türkei heimisch, wo sie in Höhenlagen zwischen 900 und $2000 \mathrm{~m}$ mit Picea orientalis vergesellschaftet ist. Sie bildet mächtige, bis $60 \mathrm{~m}$ hohe und $2 \mathrm{~m}$ starke Waldbäume. In Mitteleuropa ist der als Parkbaum beliebte Baum bis ins hohe Alter ansehnlich und kann $30 \mathrm{~m}$ hoch werden.

Hier sind zwei Individuen der Spanischen Tanne (Abies pinsapo 'Kelleriis', Wuchshöhen: 15 und $17 \mathrm{~m}$ ) anzutreffen. Die Spanische Tanne ist in kleinen Arealen in der Provinz Malaga in Südspanien und Marokko heimisch, wo sie in Höhen von 1000 bis $1500 \mathrm{~m}$ vorkommt. Sie kann 30 m Höhe erreichen bei einem Stamm- durchmesser von bis zu $1,5 \mathrm{~m}$. Die 15 bis $20 \mathrm{~mm}$ langen, stechenden Nadeln sind im oberen Kronenbereich kürzer und stehen rund um den Zweig. Sie haben einen abgeflacht viereckigen Querschnitt und sind in der Mitte der Zweige oft nach hinten gebogen. Durch die charakteristische Benadelung ist der Baum ein beliebtes Ziergehölz.

Die Weiß-Fichte (Picea glauca, geschätzte Wuchshöhe: $15 \mathrm{~m}$ ) kommt in Nordamerika von Alaska bis Neufundland und in den nördlichen USA von Tallagen bis in die Gebirge natürlich vor. Dort bildet sie große Wälder. Der Baum kann 20 bis $40 \mathrm{~m}$ hoch werden und ist ein forstwirtschaftlich wichtiger Holzlieferant. Die Äste sind ansteigend, die Zweige meist hängend. Ihre Nadeln sind 1 bis $1,8 \mathrm{~cm}$ lang, stumpf, ziemlich starr und graugrün. Die Zapfen sind kleiner als bei Picea abies, 3 bis $6 \mathrm{~cm}$ lang, bis $2 \mathrm{~cm}$ dick und im Reifezustand hellbraun gefärbt.

Drei Exemplare der Kaukasus-Fichte (Picea orientalis, Wuchshöhen: 12, 18 und $21 \mathrm{~m}$ ) sind 


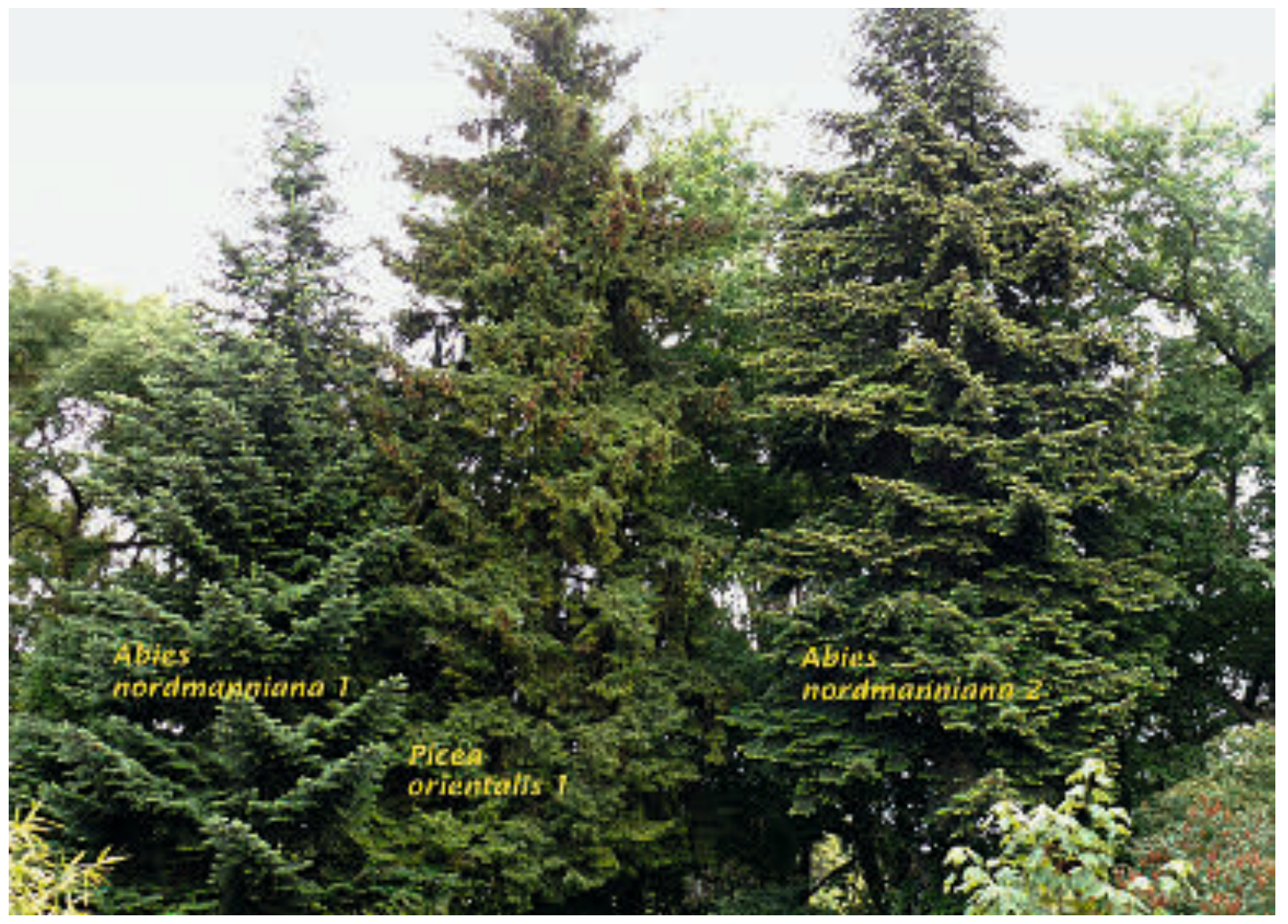

hier sehr markant. Sie kommt in Gebirgen an der Ostseite des Schwarzen Meeres zwischen 1100 und 2100 m vor. In höheren Lagen bildet die Art Reinbestände, sonst kommt sie in Mischung mit Abies nordmanniana, Fagus orientalis u. a. vor. Der Baum erreicht in seiner natürlichen Heimat bis 50 m Höhe, der Stamm kann einen Durchmesser von $1 \mathrm{~m}$ erreichen. Picea orientalis verträgt trockene Sommer. Die dicht stehenden, glänzend dunkelgrünen Nadeln sind mit $8 \mathrm{~mm}$ Länge die kürzesten aller Fichtennadeln. Im Freistand ist der Baum tief beastet.

Der Japanische Lebensbaum (Thuja standishii, Wuchshöhe: $13,50 \mathrm{~m}$ ) ist ein bis $30 \mathrm{~m}$ hoher Waldbaum aus dem Zentralgebirge Mit-

Abb. 2 (Seite 24): Blick vom Institutsgebäude auf einen Teil der Laubgehölze. Von links nach rechts: Platanus occidentalis, Tilia mongolica, Tilia $x$ vulgaris.

Abb. 3: Blick auf verschiedene Koniferen auf dem Gelände. Von links nach rechts: Abies nordmanniana, Picea orientalis, Abies nordmanniana.
tel-Japans, wo er bis in Höhen von 2000 m vorkommt. Als wichtiger Baum Japans liefert er dauerhaftes, wasserfestes, attraktiv duftendes Holz. Er gilt in Japan als einer von fünf heiligen Bäumen. Diese fünf heiligen Baumarten sind Hinoki-Scheinzypresse (Chamaecyparis obtusa), Erbsenfrüchtige Scheinzypresse (Chamaecyparis pisifera), Schirmtanne (Sciadopitys verticillata), Japanischer Lebensbaum (Thuja standishii) und Hiba-Lebensbaum (Thujopsis dolabrata). In Europa ist die Art nur vereinzelt in Arboreten zu finden.

Das natürliche Vorkommen der Salzmann's Schwarz-Kiefer (Pinus nigra subsp. salzmannii, Wuchshöhe: $6,50 \mathrm{~m}$ ) ist auf kleinere Areale in Algerien, Marokko, Spanien und Südfrankreich (Cevennen und östl. Pyrenäen) beschränkt. Diese Kiefer, die hier am Standort erst vor wenigen Jahren gepflanzt wurde, wird auch in der Natur höchstens $20 \mathrm{~m}$ hoch. Sie ist eine wenig wüchsige, standorttolerante, dürreharte und genügsame Unterart der "normalen“ Schwarz-Kiefer. 